\title{
A New Power Control Approach for IEEE 802.11 Ad Hoc Networks
}

\author{
Xiao-Hui Lin, Yu-Kwong Kwok, and Vincent K. N. Lau \\ Department of Electrical and Electronic Engineering \\ The University of Hong Kong, Pokfulam Road, Hong Kong,China
}

\begin{abstract}
In packet radio networks, especially an ad hoc wireless network using IEEE 802.11 as the MAC (multiple access control) protocol, power control is a crucial issue. By using a judicious power control mechanism, co-channel interference can be significantly reduced, thus improving the channel spatial reuse and network capacity. However, efficient power control in an IEEE 802.11 system is very challenging because according to the standard, fixed power is used for transmitting packets, and there is only one channel. In this paper, we propose an enhancement to the standard IEEE 802.11 MAC protocol by improving the handshaking mechanisms and adding one separate power control channel. With the control channel, the receiver notifies its neighbors its noise tolerance. Thus, the neighbors can adjust their transmission power levels to avoid packet collisions at the receiver. Through extensive simulations using NS-2, our proposed power control mechanism is found to be effective in that network throughput can be increased by about $10 \%$, and the battery utilization can also be improved at the same time.
\end{abstract}

Key Words: power control, ad hoc networks, IEEE 802.11, medium access control.

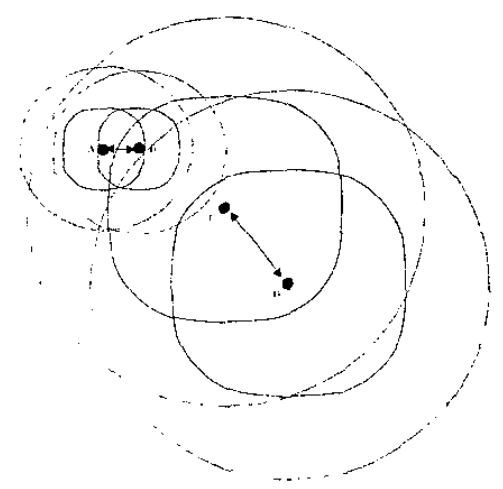

Fig. I. Asymmetrical link problem.

\section{Asymmetrical Link Phenomenon and The PROPOSED PCMAC PROTOCOL}

In our study, we consider the power control problem in an IEEE 802.11 based ad hoc network. Specifically, the data reception area in such a network can be divided into two zones: decoding zone and carrier sensing zone. In the decoding zone, the received packet can be correctly decoded. While in the sensing zone, the packet can only be sensed (i.e., signal is detected) but not decoded. The sizes of these two zones can change with the variation of transmission power level. Different transmission power levels generated by distinct mobile terminals in a fully distributed manner introduce the asymmetrical link phenomenon because, compared with the original fixed normal (maximal) power level, using different power levels reduces the decoding and carrier sensing area. When the surrounding terminals cannot decode or sense the packet (because they are outside the decoding and sensing zone), they cannot adjust their NAVs (Network Allocation Vectors), and thus, they deduce that the wireless channel is free and transmit their own packets, causing collisions. This scenario is depicted in Figure 1. We can see that there are two source and destination pairs: $\mathrm{A} \leftarrow \mathrm{B}$ and $\mathrm{C} \leftarrow \mathrm{D}$. Terminals $\mathrm{C}$ and $D$ are outside the carrier sensing zone of $A$ and $B$, and thus, $C$ cannot sense the signals sent by $A$ or $B$. C can cause packet collision problems to $B$ if C's transmission power is high enough.

In the literature, a basic power control scheme has also been suggested [5]. In this scheme, the RTS-CTS dialog uses the normal (maximal) power level, while DATA-ACK uses the minimal needed power level. This scheme is illustrated in Figure 2. As can be seen, the wireless channel is first "reserved" by RTS-CTS, and the potential terminals in the maximal decoding zone of the sender and receiver can adjust their NAVS when receiving the RTS or CTS. In this manner, the probability of packet collision is greatly reduced. However, the drop of DATA transmission power level also results in the shrink of sensing zone. When the terminals in the original sensing zone cannot sense the signal, they might consider that the channel is free and transmit their packets, thus causing packet collisions. This is also an example of asymmetrical link phenomenon. The scenario is depicted in Figure 3. The same observation and analysis can also be found in [5].

Asymmetrical link phenomenon causes the inefficient usage of the wireless channel resource, thus leading to serious consequences: (1) the frequent data collisions, re- 


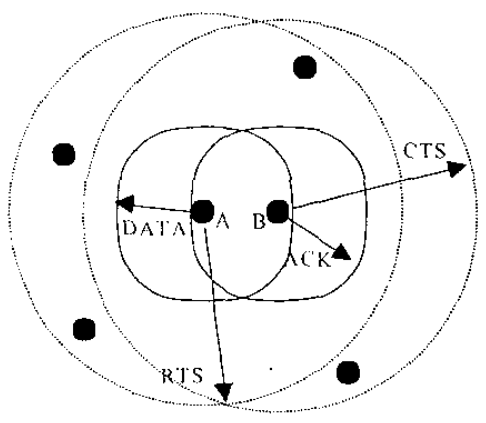

Fig. 2. RTS and CTS are transmitted at the normal power level: while DATA and ACK are transmitted at the required power level.

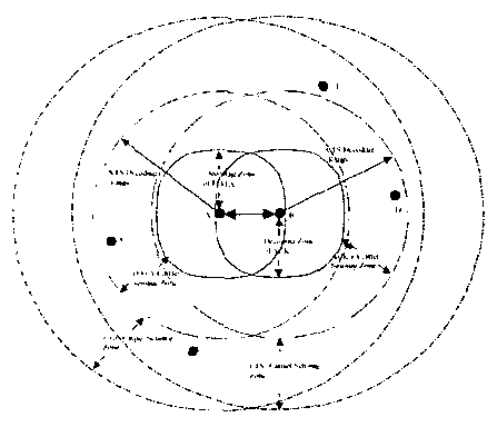

Fig. 3. Terminal A sends data packets to B. RTS and CTS are transmitted at the normal power level: while DATA and $A C K$ are transmitted at the required power level. The reduction of power level for DATA and ACK causes the shrinking of the carricr sensing zone. Thus, teminal $E$ and $F$ cannot sense the DATA and ACK. and hence, collisions can occur if $E$ and $F$ transmit at a high enough power.

sulting in more retrausmissions, in turn leading to a waste of the limited wireless bandwidth and battery power; (2) deterioration in network performance in that capacity is decreased and packet delay is prolonged; (3) unfairness in the wireless channel usage, e.g., in Figure 1, the transmission belween $\mathrm{A}$ and $\mathrm{B}$ is frequently suppressed by $\mathrm{C}$ and $D$, hetween which, a much higher power level is needed.

The challenging points of power control in an IEEE 802.11 system are: (1) eliminating the collision at both sides (DATA collision at receiver side and ACK collision at sender side), under the asymmetrical links environment; (2) eliminating the collision without sacrificing the network capacity; (3) ensuring the fairness among all senderreceiver pairs, i.e., the communication pair using higher power level should not suppress the nearby communication pair using relatively lower power level. However, most of the schemes cannot satisfy all of these possibly conflicting requirements. In this regard, we propose a new poner control medium access control (PCMAC) protocol, which can eliminate the negative effects induced by the asymmetrical links. In PCMAC, a seperate power control channel is used to avoid data packet collision at receiver side. All packets, including RTS, CTS, DATA, and ACK (if any), are transmitted at the most desirable power level.

Each terminal keeps two tables: table of sent packets and table of received packets. When a sender transmits a data packet to a particular receiver, the sender should record the session ID (session ID uniquely identifies the particular source-destination pair) and the sequence number of this packet, together with the ID of the particular receiver, in its table of sent packets. The sender also keeps a copy of this packet for any future retransmissions (if needed) Similarly, when a terminal receives a data packet. the receiver records the session ID and sequence number of the received packet, together with the ID of the sender, in its table of received packets. Before giving the detailed description of the proposed PCMAC, some assumptions are in order:

1. The power control channel has no interference on the data channel. Two channels share the same propagation characteristics, for example, have the same attenuation and fading parameters, and the transmission ranges are the same if using the same power level.

2. The propagation conditions (attenuation, fading, etc.) between source and destination terminal is assumed to be the same in both directions, or the propagation gain in both directions are the same $G_{i j}=G_{j i}$;

3. There is also collision in the power control channel. To reduce the collision probability, the length of broadcast packet should be kept short. Thus, the packet only includes the terminal ID and the noise endurance at the receiver. The packet frame structure is shown in Figure 4.

\begin{tabular}{|c|c|c|c|}
\hline 16 bits & \multicolumn{1}{c}{ xhir } & 16 bits & 8 birs \\
\hline Preamble & Nole ID & Noise Tolerance & FEC \\
\hline
\end{tabular}

Fig. 4. Frame structure of the power control packet.

4. The length of the data packet is fixed ( 512 bytes in our experiment) such that upon receiving the power control signaling messages over the control channel, other terminals know the residue duration time of this data reception. 5. The transmission power is limited by the most vulnerable neighbor (with the smallest signal noise ratio).

Now, we give a step-by-step description of PCMAC.

Step 1: When a mobile terminal $\mathrm{A}$ has a packet to be sent to terminal B, A checks whether the wireless channel is now busy by detecting the physical air interface and the NAV. If the channel is temporarily being used, it backs off and keeps monitoring the channel until it is relinquished. If the channel is free, it further looks up its power history 
table. to see at which power level it should use to communicate with terminal $\mathrm{B}$. Assume that the power level is P.ik.

Step 2: Tcrminal A computes whether using power level $P_{A 1}$ might cause collision at the nearby receivers. Essentially, such a constraint must be satisfied: for each nearby current receiver known to $\mathrm{A}$, say $\mathrm{C}$, the induced noise level at $C: G_{A C} P_{A B} \leq 0.7\left(\frac{P_{r c}}{S T R_{T h}}-P_{n c}\right)$. Here, $G_{A C}$ is the propagation gain from $\mathrm{A}$ to $\mathrm{C}$, and $G_{A C} P_{A B}$ is the induced noise by $\mathrm{A}$ at $\mathrm{C} \cdot \frac{P_{r c}}{S f \kappa_{r h}}-P_{n c}$ is the noise tolerance at $\mathrm{C}$ and is known to $A$. We choose the coefficient 0.7 because: (1) the noise level might be fluctuating at $C$ (although through our observations, this fluctuating scope is rather small in the short span of a data reception, about $2.2 \mathrm{msec}$ ); and (2) there might be other terminals also wanting to transmit at the same time. Thus, we should leave some redundancy in the noise tolerance at terminal $\mathrm{C}$. If this constraint cannot be satisfied. terminal A must back off until the reception is completed: otherwise, teminal A can send RTS out, waiting for CTS from C. This RTS also includes the noise level $P$ at terminal A and the power level $P_{\Gamma}$ at which RTS is transmitted. If timeout occurs and A cannot receive CTS from B. A increases its power level (by one step until gets to maximal level) and repeat the computation as mentioned above.

Step 3: If terminal B reccives the RTS, it should reply with CTS, which should be transmitted at the power level of ":ar:\{ $\left.R x_{T !} P_{T A} . S I R R_{T} P_{n A}\right\}$, so that this CTS can be captured and received at sender $A$. Here, $E_{B A}$ is the observed RTS receive power at $\mathrm{B}$, and $G_{A B}$ is the propagation gain which can be computed based on $P_{T A}$ and $E_{B A 1}$. In order that the following DATA from terminal A can be also captured and received at $B, B$ requires DATA be sent at the power level $\max \left\{\frac{R x_{h h} P_{T A}}{E_{B A}}, \frac{S I R_{T h} P_{n B}}{G_{A B}}\right\}$. B also puts this information into the CTS. Before transmitting CTS, teminal B must also perform the collision computation same as terminal $\mathrm{A}$, so as to avoid collision at the surrounding receivers. If $B$ is allowed to send CTS, it appends to CTS the session ID, together with the sequence number of the last data packet received from $\mathrm{A}$, then sends this CTS to A.

Step 4: When terminal A receives CTS, it compares the session ID and sequence number included in CTS with those stored in its table of sent packets, to perform a successful reception check of the last sent packet. If the two match. terminal A transmits the next data packet to $B$, and updates its table of sent packets by storing the related information of this next data packet in the table. If these two ficids do not match, terminal $A$ has to retransmit the last sent data packet to B. Before transmitting the DATA packel at the required power level, terminal $A$ again re- peats the collision avoidance computation.

Step 5: When terminal B begins to receive data packet, it estimates the signal and noise strength, computing the noise level that it can still endure by $\frac{P_{r B}}{\operatorname{Sir} R_{T h}}-P_{n B}$, and then broadcast this information out through the power control channel at the normal power level.

Step 6: If terminal B successfully receives this data packet, it updates its table of received packets by storing the session ID and sequence number in it.

Step 7: Terminal B can choose to reply A with an ACK, if the received packet is not a data packet (e.g., is a RREP or RRER), or just return to IDLE state, if the received packet is DATA.

The transmission of other unicast packets (non-data packet, such as RREP or RRER) is similar to that of a data packet, except that there is no need to have a check of last sequence number and session ID, and the receiver has to reply the sender with an ACK to confirm the successful reception.

\section{SIMULATION ENVIRONMENT AND RESULTS}

In order to test the performance of PCMAC, we use NS2 (Version ns $2.1 \mathrm{~b} 8 \mathrm{a}$ ), a discrete event simulator extended by CMU Monarch project to support ad-hoc routing, as our simulation platform. NS-2 contains a complete set of ad hoc routing protocols and can support IEEE 802.11 MAC standard that executes a wireless RF physical layer operating at $914 \mathrm{MHz}$, with a data rate of $2 \mathrm{Mbps}$. All the wireless physical layer parameters in the simulator have been tuned to model the Lucent WaveLAN card. In NS2 the decoding and sensing ranges are $250 \mathrm{~m}$ and $550 \mathrm{~m}$, respectively, when using the normal power level.

We choose the basic IEEE 802.11 without power control and two schemes with power control as our references. In Scheme 1, RTS and CTS are transmitted at the normal power level, while DATA and ACK are transmitted at the needed power level. In Scheme 2, all the packets, including RTS, CTS, DATA and ACK are transmitted at the desirable power levels. The broadcast packets are transmitted at the normal power level in all protocols, including Scheme 1 and Scheme 2, PCMAC and basic 801.11. In Scheme 1 and Scheme 2, each mobile terminal also keeps a power history table as in PCMAC, and the table updating mechanism is also similar to that of PCMAC. We choose Scheme 1 and Scheme 2 as our references because they are adopted by many other power control algorithms [1], [2], [3], [4], [9], [10], which are designed for tackling the asymmetrical link problem.

Same as the parameters used in [5], in our simulation we adopt ten transmission power levels: $1 \mathrm{~mW}, 2 \mathrm{~mW}$, $3.45 \mathrm{~mW}, 4.8 \mathrm{~mW}, 7.25 \mathrm{~mW}, 10.6 \mathrm{~mW}, 15 \mathrm{~mW}, 36.6 \mathrm{~mW}$, 
$75.8 \mathrm{~mW}$. and $281.8 \mathrm{~mW}$, which roughly correspond to the decoding range of $40 \mathrm{~m}, 60 \mathrm{~m}, 80 \mathrm{~m}, 90 \mathrm{~m}, 100 \mathrm{~m}, 110$ $\mathrm{m}, 120 \mathrm{~m}, 150 \mathrm{~m}, 180 \mathrm{~m}$, and $250 \mathrm{~m}$, respectively, when the two-way ground propagation model (see NS manual $161)$ is adopted. The simulation parameters we used are as follows:

- number of terminal: 50 ;

- testing field: $1000 \mathrm{~m} \times 1000 \mathrm{~m}$;

- mobile speed: $3 \mathrm{~m} / \mathrm{s}$;

mobility model: random way point, i.e., when the terminal reaches its destination, it pauses for 3 seconds, then randomly chooses another destination point;

bandwidth of the power control channel: $500 \mathrm{kbps}$;

- traffic model: continuous bit rate (CBR), using UDP with packet size of 512 Bytes, and 10 source and destination pairs in the network;

- simulation time: 400 seconds;

- routing protocol: AODV [7], [8], which has been implemented in NS-2.

To evaluate the four MAC protocols, we increase the trafyic load until the network get saturated, comparing them by using the following metrics:

- Aggregate Network Throughput: average number of data packets arrives at their destinations per second in the whole network scale. measured in kbps;

- Arerage End-to-End Delay: measured in msec, the endto-end delay stands for the duration time for a packet transmitted from its source to the destination:

- Control Overhead: measured in bps, control overhead includes routing overhead (e.g., RREP, RRER in the network layer), MAC layer overhead (e.g., RTS, CTS, ACK), and ARP overhead;

- Remaining Battery Power: average battery power remaining in each mobile terminal within the elapsed time, measured in Joule.

We test all the MAC protocols under a relatively low mobility environment, because our focus is on investigating how MAC protocols can influence the above mentioned metrics. instead of how the routing protocol reacts in a high mobility enviromment. High mobility might obscure the important observations and more network overhead is generated.

Figure 5 shows the increase of aggregate network throughput with the increase in traffic load. We can see that PCMAC has the highest network throughput among foul MAC protocols. By using PCMAC, the network capacity has an improvement of about $8-10 \%$, compared with that of basic IEEE 802.11 , which is an unmodified MAC protocol without power control. Adopting power control can realize wireless channel spatial reuse, thus allowing more simultaneous transmissions. This, of course, increases the network capacity. However, as discussed earlier, using power control, packet collisions due to asymmetrical link problem must be properly tackled. In Scheme 1 , the transmission of RTS-CTS is with the normal power level, but the drop in power level with DATA-ACK causes the shrink of sensing zone. Thus, terminals outside the sensing zone might cause collision at both sides, as illustrated in Figure 3. In Scheme 2, however, all nonbroadcast packets are transmitted at the needed power. This introduces more asymmetrical links, in turn causing more packet collisions than that in Scheme 1. Collision incurs the retransmission of the packet, which is a waste in the limited wireless bandwidth, thus decreasing the network capacity.

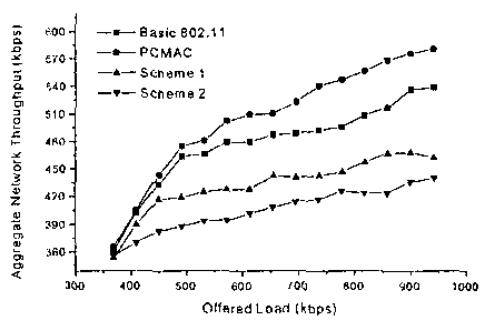

Fig. 5. Aggregate network throughput versus offered load.

Figure 6 illustrates the average packet end-to-end delay versus the increased traffic load. In all protocols, the endto-end delay increases with the load because the network gets more congested. Due to the judicious power control in PCMAC, packet delay in PCMAC is the shortest. With an appropriate power control scheme, wireless resource management is more reasonable, and channel spatial reuse decreases the packet queuing time (waiting for the availability of the channel) in its buffer, thus shortening the end-to-end delay. However, in Scheme 1 and Scheme 2, frequent packet collision incurs the retransmission of the packet, which increases the packet delay. Form the figure, the asymmetrical link problem seems more serious in Scheme 2 than in Scheme 1.

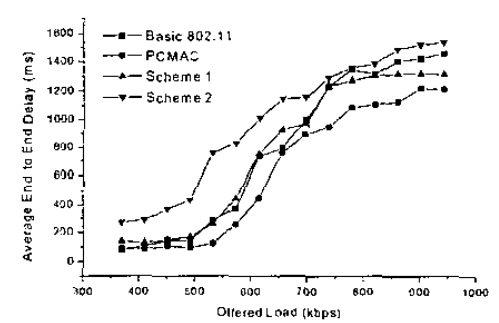

Fig. 6. Average end-to-end delay versus offered load.

Figure 7 shows the control overhead versus increased traffic load. Control overhead includes the overhead from 
the network layer, MAC layer, and ARP (address resolution protocol). Four protocols are tested under a low mobility environment, thus the overhead is mainly generated by the MAC layer to exchange data. In PCMAC, the power control packets over the control channel are also taken into account. We can see that the amount of control overhead generated by PCMAC is the least because: (1) in PCMAC. the ACK for the data packet is no longer needed, thus greatly reducing the needed overhead: (2) data collision happens not so frequently as in Scheme 1 and Scheme 2 . and this also reduces the number of data retransmission; and (3) the length of the power control packet is quite short, and it will not increase the overhead greatly. While in Scheme 1 and Scheme 2, the asymmetrical link problem incurs frequent data collisions and retransmissions, thus the control overhead is rather high. In particular, in Scheme 2, the amount of overhead is nearly twice of that generated by PCMAC or basic 802.11 .

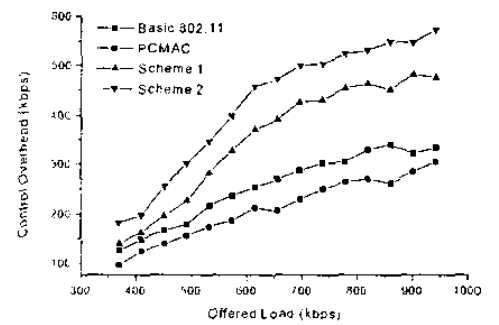

Fig. 7. Control overhead versus offered load.

In Figure 8, we further test the battery power utilization in four protocols by the average remaining power in each terminal with elapsed time. It is observed that, the power control scheme can reduce the power consumption by using only the needed power level as in PCMAC, in which, the battery can last the longest among four MAC protocols. This is very meaningful to the mobile users, who might equip with a PDA, notebook or other handset, in which the limited battery power is a precious resource. In Scheme 1 and Scheme 2, the packet retransmission is power consuming and the device might run out of power sooner.

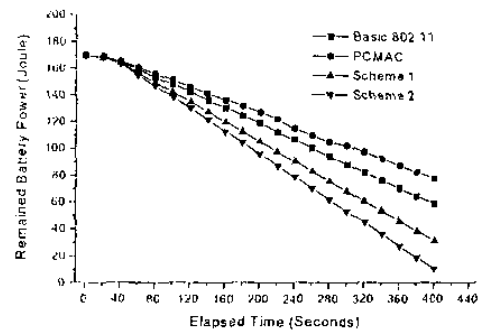

Fig. 8. Average remaining battery power against time.

\section{CONCLUSIONS}

We have presented a new power control MAC protocol, which can effectively alleviate the asymmetrical link problem. Through extensive simulations, PCMAC has demonstrated its distinctive features in that network capacity is increased and battery power utilization is improved. Furthermore, without great modifications in the firmware and software, PCMAC can be practically incorporated into the standard IEEE 802.11.

\section{REFERENCES}

[1] S. Agarwal, S. V. Krishnamurthy, R. H. Katz, and S. K. Dao. "Distributed Power Control in Ad Hoc Wircless Networks," Proc. 2001 IEEE Int'/ Symposium on Personal. Indor and Mobile Radio Communications (PIMRC'2001), pp. 59-66. Sept. 2001.

[2] P. Chen. B. O'Dea, and E. Callaway, "Energy Efficient System Design with Optimum Transmission Range for Wireless Ad Hoc Networks," Proc. ICC'2002, vol. 2, pp. 945-952, Mar. 2002.

[3] S. Doshi, S. Bhandare, and T. X. Brown. "An On-Demand Minimum Energy Routing Protocol for a Wireless Ad Hoc Network." Mobile Computing and Conmunication Review, vol. 6, no. 3, pp. 50-66, Mar. 2002.

[4] J. P. Ebert and A. Wolisz, "Combined Tuning of RF Power and Mcdium Access Control for WLAN's." Proc: 1999 IElE In'l Workshop on Mobile Multimedia Communications (MoMuC (9)), pp. 74-82, Nov. 1999.

[5] E.-S. Jung and N. H. Vaidya. "A Power Control MAC Protocol for Ad Hoc Networks." Proc: ACM 8 th Annual Intemational Conference on Mobile Computing and Networking (MOB/COM'2002). pp. 36-47, Sept. 2002.

[6] The Network Simulator (NS-2), http://ww.isiedu/nsnam/ns/, 2002.

[7] C. E. Perkins, Ad Hoc: Netnorking. Addison-Wesley, 2001.

[8] C. E. Perkins and E. M. Royer. "Ad-hoc On-Dcmand Distance Vector Routing, Mobile Computing Systems and Applications." Proc: WMCSA 99, pp. 90-100, 1999)

[9] I. Stojmenovic and X. Lin. "Power-Awarc Localized Routing in Wireless Networks," ILEE Trans. Parallel and Distributed Systems, vol. 12, no. 11, pp. 1122-1133, Nov, 2001.

[10] R. Wattenhofer, L. Li, P. Bahi, and Y.-M. Wang, "Distributed Topology Control for Power Efficient Operation in Multihop Wireless Ad Hoc Networks." Proc. INFOCOM 2001, pp. 404413, Apr. 2001 